\title{
From fusion hindrance to oscillations
}

\author{
G. Montagnoli, ${ }^{1, a}$ \\ ${ }^{1}$ Dipartimento di Fisica e Astronomia, Università di Padova, and INFN, Sezione di Padova, I-35131 Padova, Italy
}

\begin{abstract}
This paper is devoted mainly to the fusion hindrance phenomenon, in its various aspects. Recent experimental results on medium-light systems where the fusion $Q$-value is positive are discussed. The application of the coupled-channels model using a shallow ion-ion potential is illustrated for ${ }^{32,36} \mathrm{~S}+{ }^{48} \mathrm{Ca}$. The detailed influence of nuclear structure on the low-energy fusion cross sections is shown for the pair of systems ${ }^{48} \mathrm{Ti}+{ }^{58} \mathrm{Fe}$ and ${ }^{58} \mathrm{Ni}+{ }^{54} \mathrm{Fe}$, where the low-lying quadrupole modes have a different degree of collectivity. The sub-barrier excitation function of ${ }^{48} \mathrm{Ti}+{ }^{58} \mathrm{Fe}$ is much larger than for ${ }^{58} \mathrm{Ni}+{ }^{54} \mathrm{Fe}$. The lighter symmetric system ${ }^{28} \mathrm{Si}+{ }^{28} \mathrm{Si}$ has been the object of recent experimental investigations. Its fusion cross sections have been measured in a wide energy range down to $\leq 1 \mu \mathrm{b}$. Above the barrier, we have a clear indication of oscillations in the excitation function, probably due to the penetration of successive centrifugal barriers, that are in rather good agreement with previous calculations. The CC model using the shallow $\mathrm{M} 3 \mathrm{Y}+$ repulsion potential is able to reproduce also the sub-barrier part of the excitation function of ${ }^{28} \mathrm{Si}+{ }^{28} \mathrm{Si}$.
\end{abstract}

\section{Introduction}

The hindrance of heavy-ion fusion process at deep subbarrier energies has been observed for several systems [1$3]$ in the last decade. The trend of the low-energy excitation functions is conveniently represented by the logarithmic derivative of the energy-weighted cross section $L(E)$. Indeed, the onset of fusion hindrance has often been associated with the energy where $\mathrm{L}(\mathrm{E})$ reaches the value (named $\mathrm{L}_{C S}$ ) expected for a constant astrophysical $S$ factor. At that energy the $S$-factor develops a maximum as a function of the energy. However in several cases the hindrance effect is not strong enough to produce an $S$-factor maximum. Then identifying its onset requires a comparison of the experimental data to standard coupled-channels (CC) calculations.

A rich systematics is now available, but the phenomenon is very intriguing and far from being fully understood. We are not yet able to give conclusive answers to some relatively simple questions. For instance, is really the fusion hindrance phenomenon a general one, and does it occur systematically also in all systems with positive fusion $Q$-value? How (quantitatively) does the structure of the two colliding nuclei and, possibly, couplings to transfer channels, affect the energy threshold below which hindrance shows up? And also, what is the basic mechanism underlying the experimental evidences?

In the next few pages some of these issues are considered, and selected examples of our present understanding of the situation will be illustrated. Subsequently, the recent data obtained for ${ }^{28} \mathrm{Si}+{ }^{28} \mathrm{Si}$ will be reported, as well as the effort to describe within the same theoretical model

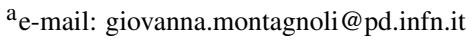

the phenomenon of sub-barrier fusion hindrance and the high energy oscillations that we observe in this system.

\section{Fusion hindrance in selected cases and theoretical interpretation}

One of the interesting features of low-energy fusion dynamics is that it is not necessary to have an S-factor maximum for systems with positive fusion $Q$-value, since finite cross section can still be expected at zero incident energy from the energy conservation law [2]. The first observation, that hindrance appears also in systems with positive $Q$-values, was obtained in a systematic study of mediumlight systems. In this region the fusion $Q$-value changes very much from system to system, being either negative or positive. With the aim to investigate the role of the $Q$-value, we recently performed a series of measurements with $\mathrm{S}$ and Ca projectiles for ${ }^{40,48} \mathrm{Ca}+{ }^{40,48} \mathrm{Ca}[4-6],{ }^{32,36} \mathrm{~S}$ $+{ }^{48} \mathrm{Ca}[7,8]$, and ${ }^{36} \mathrm{~S}+{ }^{64} \mathrm{Ni}[9]$.

A collection of six $S(E)$ are shown in Fig. 1. The blue dash-dotted curves in Fig. 1 are calculated with a standard $\mathrm{CC}$ model. These calculations overpredict the data for all systems except for the fusion of ${ }^{32} \mathrm{~S}+{ }^{48} \mathrm{Ca}$ [7], showing that the hindrance phenomenon is present, even if a $S$ factor maximum has not been reached. In the system ${ }^{40} \mathrm{Ca}$ $+{ }^{48} \mathrm{Ca}$, which has a positive $Q$-value, an $S$ factor maximum has anyway been observed. It is clear that more data points at even lower energies would be desirable to make a firm conclusion in this case. The red arrows indicate upper limits $E_{s}^{e m p}$ for the onset of fusion hindrance according to the phenomenological systemtics of Jiang [10].

To date, these (and other) studies evidence that fusion hindrance is a general phenomenon (with some exceptions 
such as ${ }^{32} \mathrm{~S}+{ }^{48} \mathrm{Ca}$ [7]) and there is no obvious dependence on the sign of the fusion $Q$-value. Therefore, if hindrance does indeed occur in light systems, such as ${ }^{12} \mathrm{C}+$ ${ }^{12} \mathrm{C},{ }^{12} \mathrm{C}+{ }^{16} \mathrm{O},{ }^{16} \mathrm{O}+{ }^{16} \mathrm{O}$, and others, the predicted rates of astrophysical processes, crucial for a variety of stellar environments, will be strongly affected.

The studies of fusion cross sections at deep sub-barrier energies, where coupling effects to the intrinsic modes of excitation should be damped, allow to probe the interaction potential itself. When the densities of two reacting nuclei overlap a description in terms of a two-body potential may fail, even when coupled-channels effects are included.

However, in order to describe the fusion hindrance phenomenon, Misicu and Esbensen [11] (capitalizing on a previous work of Dasso and Pollarolo [12]) have developed a model using an ion-ion potential adapted to overlapping nuclei using realistic densities. The potential is obtained by double folding and uses the effective M3Y nucleon-nucleon interaction supplemented by a repulsive contact term taking into account the nuclear matter incompressibility (M3Y + rep). The result is a shallow potential that produces a thicker barrier in the entrance channel, when compared to a standard Woods-Saxon (WS) potential.

As an example, Fig. 2 shows the measured fusion excitation functions of two systems ${ }^{32,36} \mathrm{~S}+{ }^{48} \mathrm{Ca}[7,8]$ compared to the CC calculations obtained within the M3Y+rep model. The calculations give a good account of the data. They suggest the influence of one- and two-nucleon transfer channels in ${ }^{32} \mathrm{~S}+{ }^{48} \mathrm{Ca}$ for which it is evident that the cross sections decrease very smoothly below the barrier.

The fusion data for ${ }^{36} \mathrm{~S}+{ }^{48} \mathrm{Ca}$ [8] can be reproduced very well by considering couplings to low-lying states and mutual excitations of these states in projectile and target. The hindrance phenomenon is observed for ${ }^{36} \mathrm{~S}+{ }^{48} \mathrm{Ca}$; however, this is not true for ${ }^{32} \mathrm{~S}+{ }^{48} \mathrm{Ca}$, where the data are well reproduced also by $\mathrm{CC}$ calculations employing a standard WS potential of the Akyüz-Winther type [13]. This is probably due to the concurring influence of transfer couplings.

Starting from different points of view other theoretical approaches have been developed [3, 14-18] that are able to reproduce various features of hindrance. Some of these models are discussed elsewhere in this Conference.

\section{Specific influence of nuclear structure}

Collective vibrations are known in several nuclei in the mass region $\mathrm{A} \simeq 40-50$, although not so strong as around $\mathrm{A} \simeq 100$. Fusion between such nuclei with well-known collective excitations at low energies may help clarifying the influence of such inelastic states on fusion and understanding the physics underlying deep sub-barrier fusion.

The system ${ }^{48} \mathrm{Ti}+{ }^{58} \mathrm{Fe}$ is appealing in this sense, since both nuclei have a low-lying quadrupole excitation: the $2^{+}$ states lie at $\approx 800-900 \mathrm{keV}$ only, while the octupole states are rather high. The behavior of this system can immediately be contrasted with the results obtained for the nearby case ${ }^{58} \mathrm{Ni}+{ }^{54} \mathrm{Fe}$ [19] (closed-shell nuclei), where the

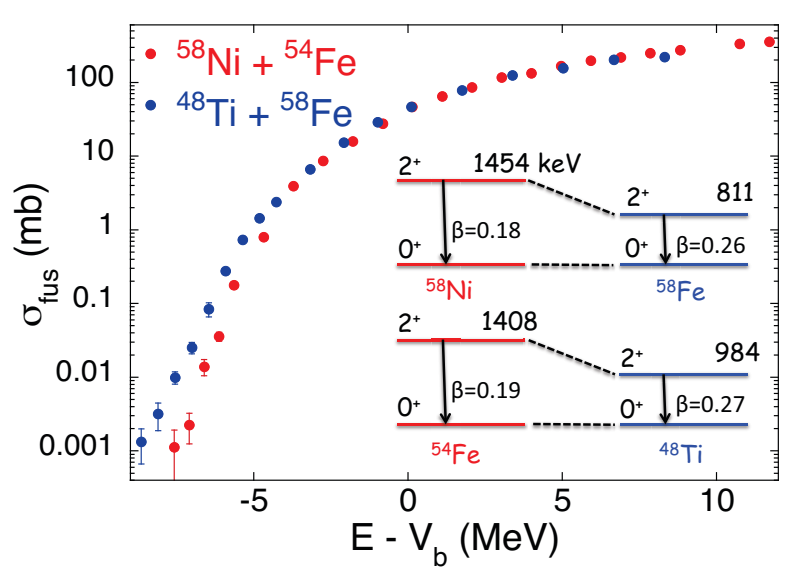

Figure 3. Fusion excitation functions of ${ }^{48} \mathrm{Ti}+{ }^{58} \mathrm{Fe}$ and ${ }^{58} \mathrm{Ni}+$ ${ }^{54} \mathrm{Fe}$ systems.
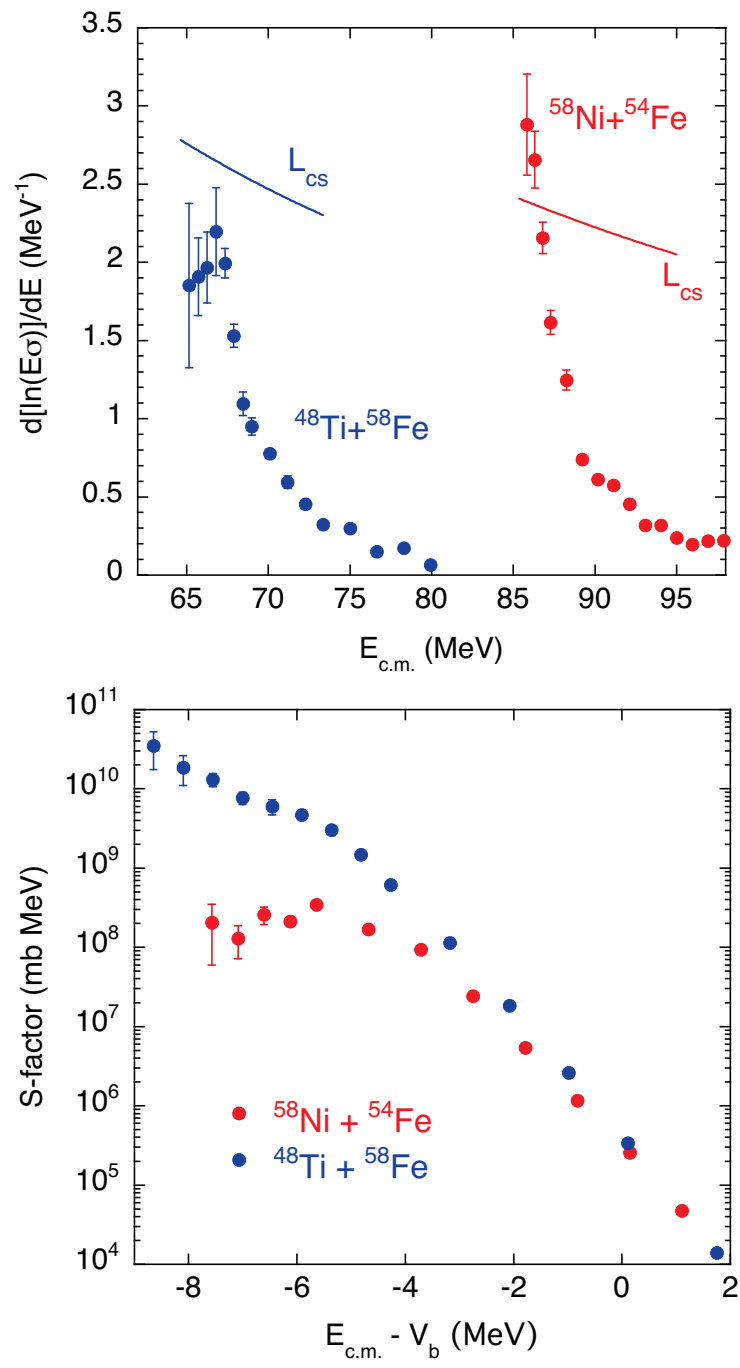

Figure 4. (top) Logarithmic derivative and (bottom) $S$-factor of the two systems. 

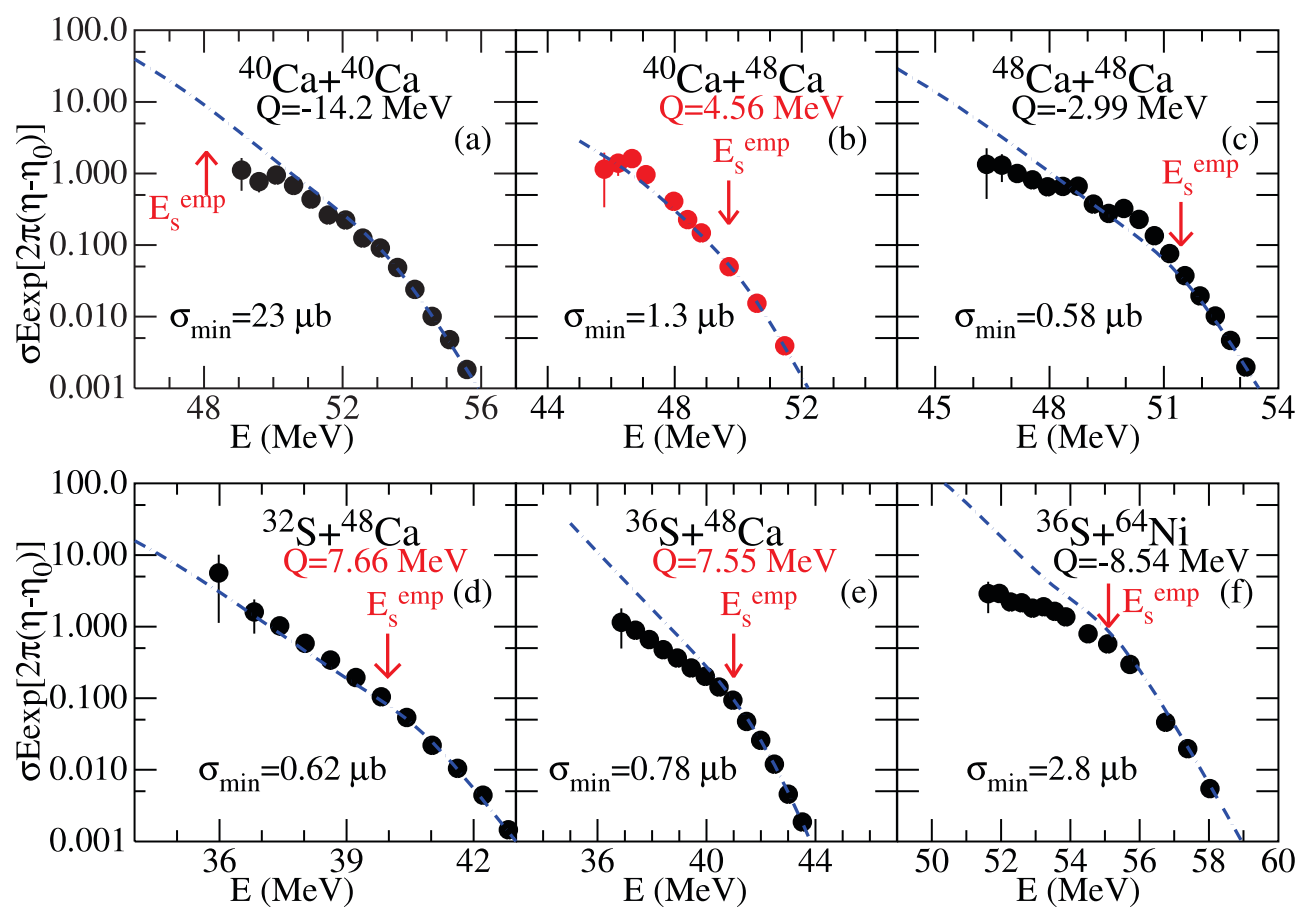

Figure 1. The astrophysical $S$-factor vs. energy for some $\mathrm{Ca}+\mathrm{Ca}$ and $\mathrm{S}+\mathrm{Ca}$ systems with different fusion $Q$-values.
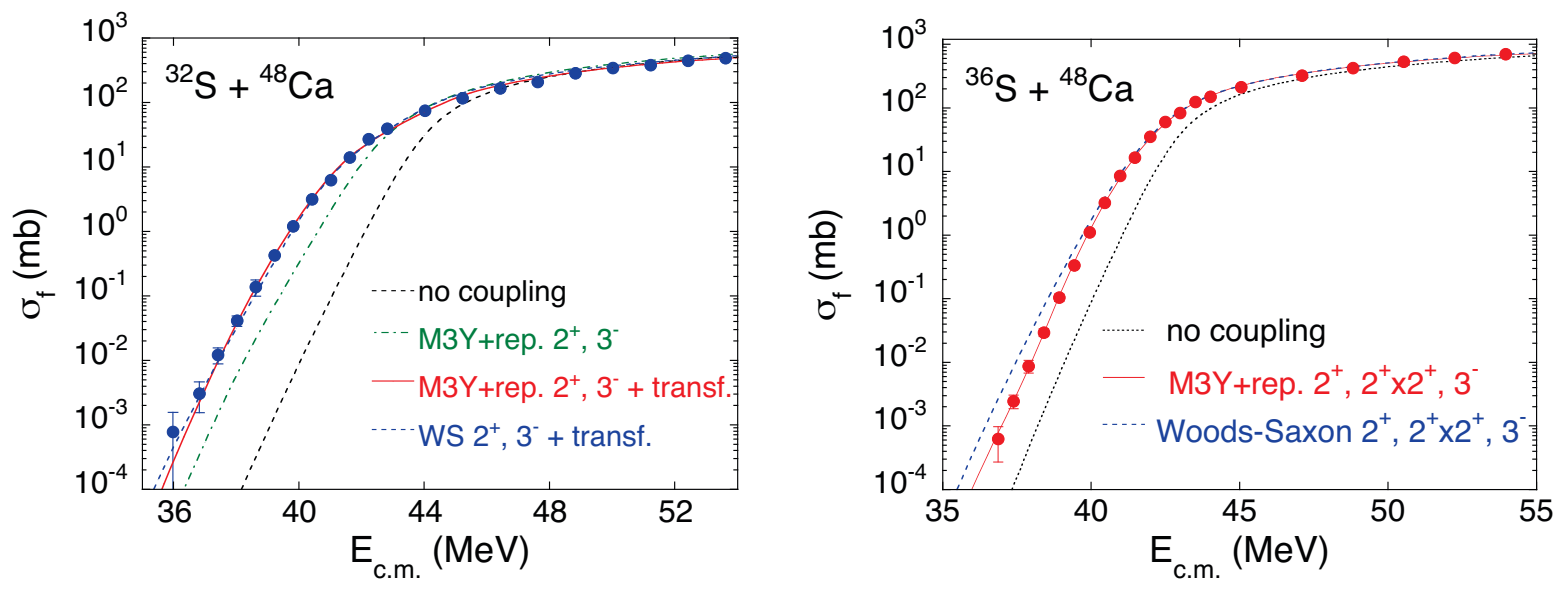

Figure 2. Experimental fusion excitation functions of ${ }^{32} \mathrm{~S}+{ }^{48} \mathrm{Ca}$ and ${ }^{36} \mathrm{~S}+{ }^{48} \mathrm{Ca}$ are compared to CC calculations [7, 8] based on a WS potential, and on the M3Y+repulsion potential.The no-coupling limit is also shown. For ${ }^{32} \mathrm{~S}+{ }^{48} \mathrm{Ca}$ the $\mathrm{CC}$ calculation also includes nucleon transfer channels (online red curve)

hindrance effect is very clear and sets in at the level of the relatively large cross section of $\sim 200 \mu \mathrm{b}$. As a matter of fact, it was observed that the cross sections decrease very steeply at the lowest energies, and the logarithmic slope of the excitation function keeps increasing, reaches and overcomes the value $\mathrm{L}_{C S}$ expected for a constant astrophysical $\mathrm{S}$ factor. Consequently, this shows a clear maximum as a function of the energy.

The measured excitation function of ${ }^{48} \mathrm{Ti}+{ }^{58} \mathrm{Fe}$ is reported in Fig. 3. It is plotted vs. the energy difference from the Coulomb barrier produced by the potential used in the $\mathrm{CC}$ calculations discussed in Ref. [20]. In the same fig- ure, also the published cross sections for ${ }^{58} \mathrm{Ni}+{ }^{54} \mathrm{Fe}$ [19] are shown in the corresponding energy scale. This allows us to notice immediately the large enhancement of ${ }^{48} \mathrm{Ti}+$ ${ }^{58} \mathrm{Fe}$ fusion with respect to the other, more stiff, system.

The insert in the same figure shows pictorially the noticeable difference of nuclear structure between the two pair of nuclei. The excitation energies and deformation parameters of the low-lying quadrupole excitations are reported.

The logarithmic derivatives (slopes) of the excitation functions for the two systems are plotted in Fig. 4 (top panel). Here one can even better appreciate the different 

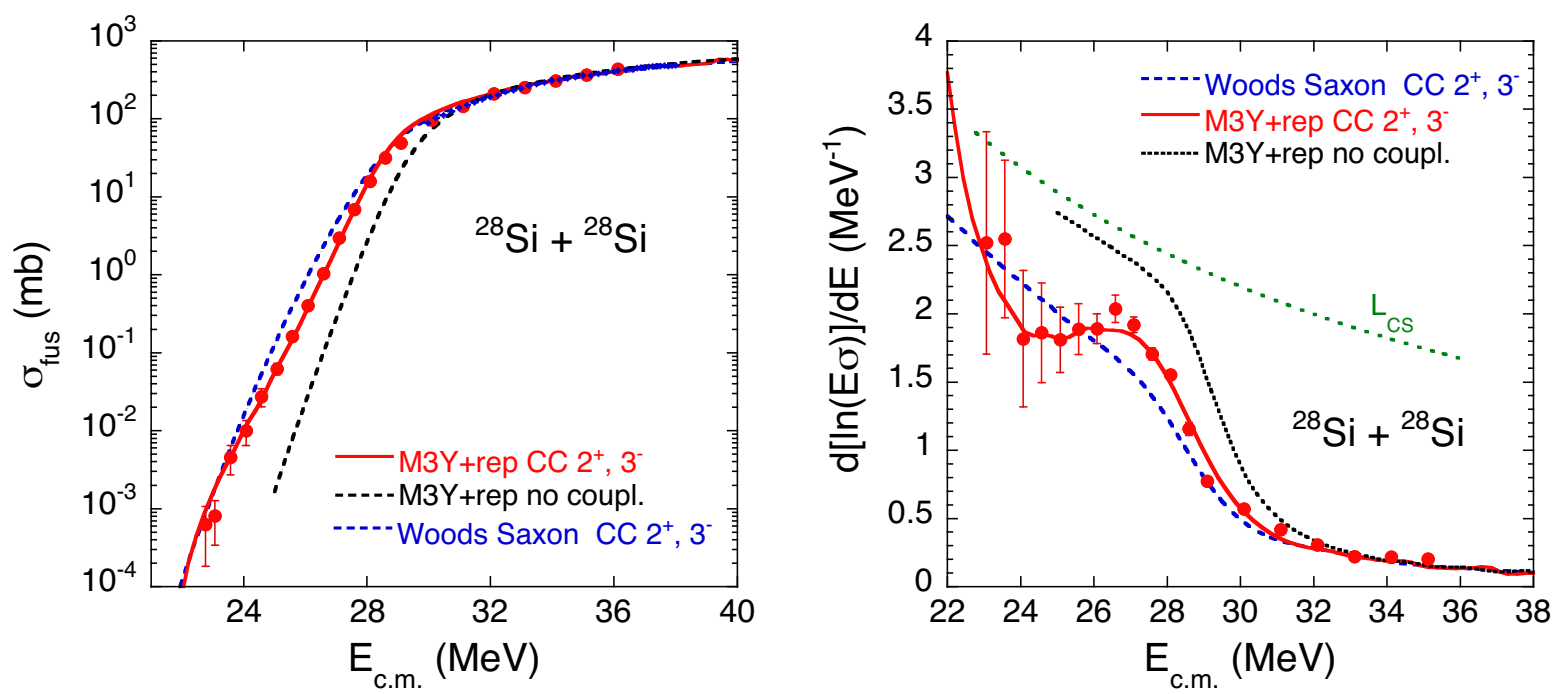

Figure 5. Fusion excitation function of ${ }^{28} \mathrm{Si}+{ }^{28} \mathrm{Si}$ (left), and its logarithmic derivative (right), compared to the CC calculations. The red line is the calculation including two phonons of the $2^{+}$state. $\mathrm{L}_{C S}$ (black dots) is the slope expected for a constant astrophysical $\mathrm{S}$ factor.

trends. $\mathrm{L}_{c s}$ is the slope expected for a constant astrophysical S factor. The slope of ${ }^{58} \mathrm{Ni}+{ }^{54} \mathrm{Fe}$ increases steadily below the barrier and overcomes the $\mathrm{L}_{c s}$ value, while the slope of ${ }^{48} \mathrm{Ti}+{ }^{58} \mathrm{Fe}$ flattens out well below that value.

The clear-cut difference between the sub-barrier behavior of the two systems can be seen in Fig. 4 (bottom panel) from a different point of view. The nice $S$ factor maximum that develops with decreasing energy for ${ }^{58} \mathrm{Ni}$ $+{ }^{54} \mathrm{Fe}$, is not observed at all for ${ }^{48} \mathrm{Ti}+{ }^{58} \mathrm{Fe}$ down to the lowest measured cross sections. The strong quadrupole modes of both ${ }^{48} \mathrm{Ti}$ and ${ }^{58} \mathrm{Fe}$ are likely to be responsible for all this. We have no indication of hindrance for ${ }^{48} \mathrm{Ti}$ $+{ }^{58} \mathrm{Fe}$ down to $\sigma_{\text {fus }} \simeq 1 \mu \mathrm{b}$. It appears that the hindrance threshold is pushed to even lower energies in this system. Coupled-channels calculations will help understanding the fusion dynamics in this interesting case.

\section{High energy structures of fusion excitation functions}

Oscillatory structures have been observed since a long time in the fusion excitation function of light heavy- ion systems like ${ }^{12} \mathrm{C}+{ }^{12} \mathrm{C},{ }^{12} \mathrm{C}+{ }^{12} \mathrm{O}$ and ${ }^{16} \mathrm{O}+{ }^{16} \mathrm{O}$ [21$24]$, in the energy region above the Coulomb barrier. Recent theoretical works $[25,26]$ have been dedicated to this subject suggesting that they may be due to the penetration of successive centrifugal barriers. In Ref. [25] it has been pointed out that those structures can be best revealed by plotting the first (energy-wighted) derivative of the excitation function. Indeed, in those light systems, the separation between nearby barriers is large with respect to the intrinsic energy width associated with their quantal penetration, so that the oscillations become observable. It seems that a shallow potential [11] is required for fitting sub-barrier as well as above-barrier cross sections, because

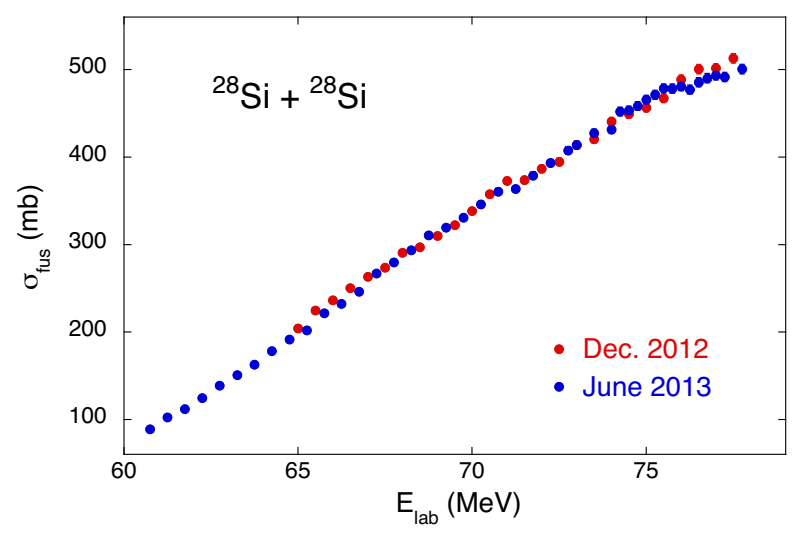

Figure 6. Fusion excitation function of ${ }^{28} \mathrm{Si}+{ }^{28} \mathrm{Si}$ in the energy region above the barrier.

the oscillations are not reproduced by using a conventional Woods-Saxon potential.

Hindrance effects are stronger and more clearly observed in heavier systems, hence a careful search for oscillations in selected cases is very attractive, this despite the difficulties due to the successive L-barriers getting closer to each other, and to the decreasing amplitude of the oscillating structures, as a consequence of couplings to several reaction channels. Very recently the system ${ }^{28} \mathrm{Si}+{ }^{28} \mathrm{Si}$ has been studied, by measuring the excitation function in a wide energy range from deep sub-barrier energies to above the Coulomb barrier with the aim to describe in the same theoretical frame its whole trend. Concerning the behaviour above the barrier, previous existing data [27] did not allow clear-cut conclusions for possible oscillations due to the large errors and to the too wide energy steps. 


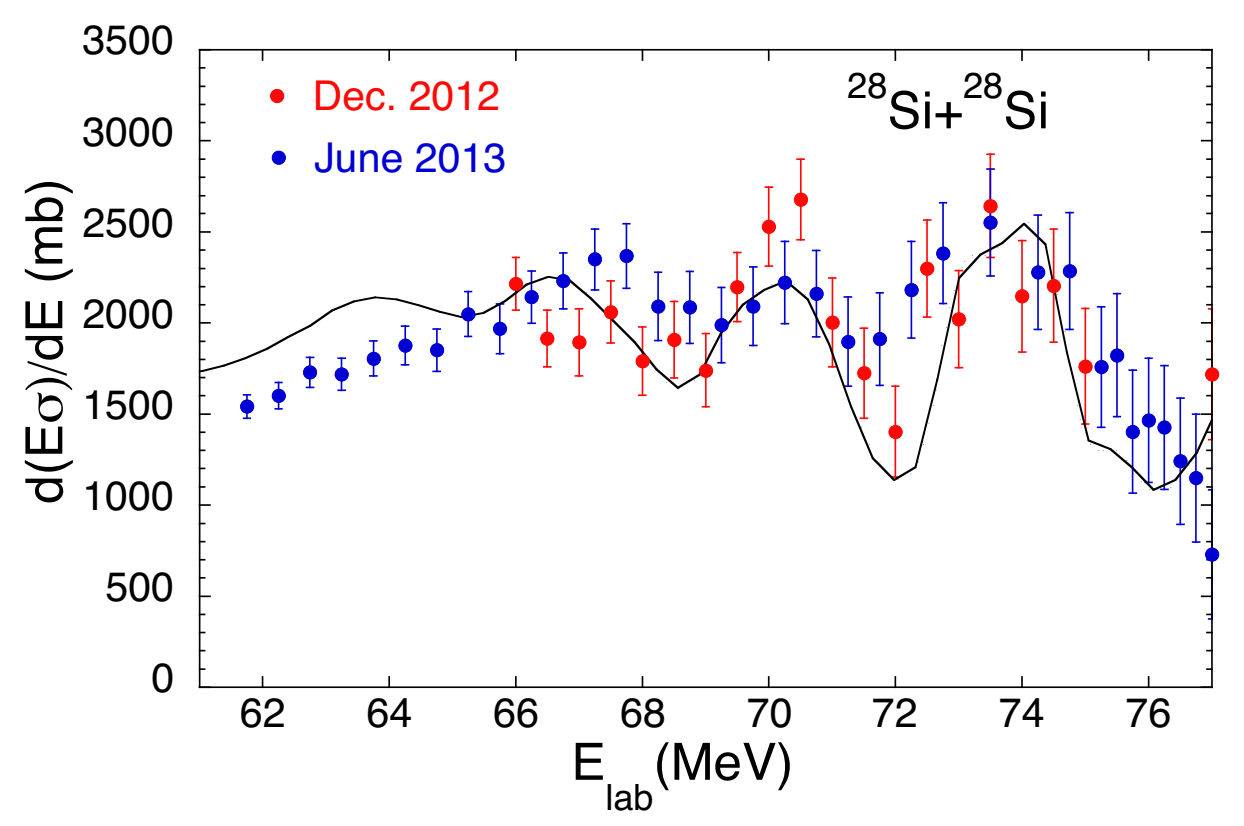

Figure 7. Energy-weighted derivative of the fusion excitation function of ${ }^{28} \mathrm{Si}+{ }^{28} \mathrm{Si}$ in an energy range above the barrier. The full line is the theoretical calculation of Ref. [25].

This system is symmetric, therefore only even values of L contribute to fusion. This is an advantage, because the peaks are more distant from each other. The distance between two successive peaks, as estimated in Ref. [25] and on the basis of the old data is $\simeq 3 \mathrm{MeV}$ in the lab system, while the FWHM of the peaks is $\approx 2.2 \mathrm{MeV}$ at $\mathrm{E}_{c m} \simeq 35$ $\mathrm{MeV}$ for $\mathrm{L}=20$.

The fusion excitation function has been measured down to very low energies [28] and the full excitation function is shown in Fig. 5 (left panel). A CC analysis of the sub-barrier excitation function has been performed, using the $\mathrm{M} 3 \mathrm{Y}+$ rep potential. The collective $2^{+}$state of ${ }^{28} \mathrm{Si}$ at $\mathrm{E}_{x}=1.779 \mathrm{MeV}$ has been coupled in, as well as the $3^{-}$octupole state at $6.879 \mathrm{MeV}$. The resulting excitation function is a very good fit of the data. The logarithmic derivative of the excitation function (right panel) is also reproduced by the calculation based on a shallow potential remarkably well. This is not the case when $\mathrm{CC}$ calculations using a conventional WS potential are performed (see again the two panels of Fig. 5).

Two different series of careful measurements above the barrier have been performed, with an energy step small enough $\left(\Delta \mathrm{E}_{l a b}=0.5 \mathrm{MeV}\right)$ to resolve possible oscillatory structures, if any. The ${ }^{28} \mathrm{Si}$ beam has been provided with high quality and precision by the XTU Tandem accelerator of the Laboratori Nazionali di Legnaro (LNL) of INFN, in the energy range $\simeq 62-78 \mathrm{MeV}$. The targets were $50 \mu \mathrm{g} / \mathrm{cm}^{2}$ evaporations of ${ }^{28} \mathrm{Si}$ onto $15 \mu \mathrm{g} / \mathrm{cm}^{2}$ carbon backings. The fusion-evaporation residues (ER) were separated from the beam by using the set-up based on an electrostatic deflector (see [4] and Refs. therein), which is systematically employed for sub- and near-barrier fusion measurements at LNL. The set-up is very simple to operate, allowing fast and reliable measurements of relative and absolute cross sections.

For each energy, at least 10000 ER were detected, thus reducing the statistical error to $1 \%$ or less.

The measured excitation function appears to be very smooth at first sight (see Fig. 6), but a closer inspection reveals small irregularities. In the figure, the statistical error bars are smaller than the symbol size and the red and blue dots refer to the two different experiments of 2012 and 2013. By extracting the energy-weighted derivative we obtain rather regular oscillations as shown in Fig. 7, although the uncertainties are obviously larger. Two well defined peaks definitely show up in both series of measurements while a third one at $\mathrm{E}_{l a b} \simeq 67-68 \mathrm{MeV}$ is only evident in the more recent run.

The black line in Fig. 7 is the $\mathrm{CC}$ prediction of Ref. [25] where the peak at around $70 \mathrm{MeV}$ corresponds to $\mathrm{L}=20$. The experimental oscillations are also reproduced remarkably well by the calculation. It is necessary to point out that the theoretical analysis of the whole set of data for this system (sub-barrier excitation function and oscillatory structures above the barrier) is still in progress. Actually, the parameters of the M3Y+rep potential used to describe the excitation function at deep sub-barrier energies are slightly different from those employed in Ref. [25] predicting the oscillations.

\section{Summary}

Measurements of fusion cross sections in the far subbarrier energy region are very challenging from the experimental point of view, but the results one can obtain have a far-reaching value for our understanding of fusion dynam- 
ics and for its close relation to the nuclear structure of the colliding nuclei.

A short survey has been given here on the present understanding of the fusion hindrance phenomenon, with particular emphasis on expectations and recent experimental results on medium-light systems where the fusion $Q$ value is positive. Two examples of the application of the CC model using shallow M3Y + repulsion potentials have been illustrated. They deal with the different behavior of the two systems ${ }^{32,36} \mathrm{~S}+{ }^{48} \mathrm{Ca}$ that were studied at Legnaro in recent years.

A typical case where the detailed influence of nuclear structure on the low-energy fusion yields shows up clearly, is the result of very recent experiment on ${ }^{48} \mathrm{Ti}+{ }^{58} \mathrm{Fe}$. The fusion excitation function has been presented, and a significant irregularity of its logarithmic slope has been observed below the Coulomb barrier, but no evidence of hindrance shows up in the measured energy range. A comparison has been done with the near-by system ${ }^{58} \mathrm{Ni}+{ }^{54} \mathrm{Fe}$ where the fusion hindrance appears at relatively high cross sections. The sub-barrier excitation function of ${ }^{48} \mathrm{Ti}+{ }^{58} \mathrm{Fe}$ is much larger than what was measured for ${ }^{58} \mathrm{Ni}+{ }^{54} \mathrm{Fe}$, where the logarithmic slope increases steadily below the barrier. The observed differences between the two systems are most likely due to the stronger and lower-lying quadrupole modes of the two nuclei ${ }^{48} \mathrm{Ti}$ and ${ }^{58} \mathrm{Fe}$.

In the last part of the talk, the case of the lighter symmetric system ${ }^{28} \mathrm{Si}+{ }^{28} \mathrm{Si}$ has been considered. Its fusion cross sections have been measured in a wide energy range down to $\leq 1 \mu \mathrm{b}$. The CC analysis using the shallow M3Y+ repulsion potential gives a good agreement with near- and sub-barrier data. Above the barrier, we have a clear indication of oscillations in the excitation function, probably due to the penetration of successive centrifugal barriers. The observed oscillations are in rather good agreement with previous CC calculations [25]. The full comparison of the results of this experiment from the deep sub-barrier region up to where the oscillations start showing up, will allow us to check the consistency of the ion-ion potential in a very wide energy range.

I am pleased to thank C.L. Jiang and A.M. Stefanini for several fruitful discussions, and $\mathrm{H}$. Esbensen for allowing me to show the results of his calculations for ${ }^{28} \mathrm{Si}$ $+{ }^{28} \mathrm{Si}$ prior to publication, and all other colleagues collaborating in the fusion experiments at LNL. The research leading to these results has received funding from the European Union Seventh Framework Programme FP7/20072013 under Grant Agreement No. 262010 - ENSAR.

\section{References}

[1] C. L. Jiang et al., Phys. Rev. Lett. 89, 052701 (2002)

[2] C. L. Jiang, B. B. Back, H. Esbensen, R. V. F. Janssens, and K. E. Rehm, Phys. Rev. C 73, 014613 (2006)
[3] M. Dasgupta, D. J. Hinde, A. Diaz-Torres, B. Bouriquet, Catherine I. Low, G. J. Milburn, and J. O. Newton, Phys. Rev. Lett. 99, 192701 (2007)

[4] G. Montagnoli, et al., Phys. Rev. C 85, 024607 (2012)

[5] C. L. Jiang et al., Phys. Rev. C 82, 041601(R) (2010)

[6] A. M. Stefanini et al., Phys. Lett. B 679, 95 (2009)

[7] G. Montagnoli et al., Phys. Rev. C 87, 014611 (2013)

[8] A. M. Stefanini et al., Phys. Rev. C 78, 044607 (2008)

[9] G. Montagnoli et al., Phys. Rev. C 82, 064609 (2010)

[10] C. L. Jiang, et al., Phys. Rev. C 75, 015803 (2007); Phys. Rev. C 79, 044601 (2009)

[11] Ş. Mişicu and H. Esbensen, Phys. Rev. Lett. 96, 11270 (2006); Phys. Rev. C 75, 034606 (2007)

[12] C. H. Dasso and G. Pollarolo, Phys. Rev. C 68, 054604 (2003)

[13] Ö. Akyüz and A. Winther, in Nuclear Structure and Heavy-Ion Physics, Proc. Int. School of Physics "Enrico Fermi", Course LXXVII, Varenna, edited by R. A. Broglia and R. A. Ricci (North Holland, Amsterdam, 1981)

[14] T. Ichikawa, K. Hagino, and A. Iwamoto, Phys. Rev. C 75, 057603 (2007)

[15] T. Ichikawa, K. Hagino, and A. Iwamoto, Phys. Rev. Lett. 103, 202701 (2009)

[16] A. Diaz-Torres, D. J. Hinde, M. Dasgupta, G. J. Milburn, and J. A. Tostevin, Phys. Rev. C 78, 064604 (2008)

[17] V. V. Sargsyan, G. G. Adamian, N. V. Antonenko, W. Scheid and H. Q. Zhang, Phys. Rev. C 85, 024616 (2012)

[18] R. Keser, A. S. Umar and V. E. Oberacker Phys. Rev. C 85, 044606 (2012)

[19] A. M. Stefanini et al., Phys. Rev. C82, 014614 (2010)

[20] A. M. Stefanini et al., contribution to this Conference

[21] P. Sperr, T. H. Braid, Y. Eisen, D. G. Kovar, F. W. Prosser Jr., J. P. Schiffer, S. L. Tabor, and S. E. Vigdor, Phys. Rev. Lett. 37, 321 (1976)

[22] P. Sperr, S. E. Vigdor, Y. Eisen, W. Henning, D. G. Kovar, T. R. Ophel, and B. Zeidman, Phys. Rev. Lett. 36, 405 (1976)

[23] D. G. Kovar et al., Phys. Rev. C 20, 1305 (1979)

[24] I. Tserruya, Y. Eisen, D. Pelte, A. Gavron, H. Oeschler, D. Berndt, and H. L. Harney, Phys. Rev. C 18, 1688 (1978)

[25] H. Esbensen, Phys. Rev. C 85, 064811 (2012)

[26] C. Simenel, R. Keser, A. S. Umar and V. E. Oberacker, Phys. Rev. C 88, 024617 (2013)

[27] S. Gary and C. Volant, Phys. Rev. C 25, 1877 (1982); Y. Nagashima et al., Phys. Rev. C 33, 176 (1986)

[28] A. M. Stefanini et al., Proc. Int. Conf. on Nucl. Phys., Florence 2013, EPJ Web of Conf. 66, 03082 (2014) 